\title{
Study on Speed Measurement of Brushless DC Motor
}

\author{
Kan Fenglong* \\ Information and Control Engineering Academy, \\ Shenyang Jianzhu University \\ Shen Yang, China \\ e-mail: arnold0110@sina.com \\ * Corresponding Author \\ Han Zhonghua \\ School of Information \& Control Engineering \\ Shenyang Jianzhu University \\ Shenyang China \\ Wang Changtao \\ School of Information \& Control Engineering \\ Shenyang Jianzhu University \\ Shenyang China \\ Wang Xin \\ School of Information \& Control Engineering \\ Shenyang Jianzhu University \\ Shenyang China
}

\begin{abstract}
Objective: The speed fluctuation of the Holzer sensor of Brushless DC motor is often compared with the speed fluctuation. which often can not be used as the feedback signal of controller. In this paper, we try to resolve this problem. Methods : The method of frequency multiplication is improved, and a new method is adopted to measure the speed of the motor. Results: we propose a solution to this problem,the problem of the instability of the original velocity measurement method is improved, which can solve the problem of velocity fluctuation. Conclusion:A new method of frequency multiplication is proposed. The control precision was $0.25 \%$ and $1 \%$ respectively. The method is proved to be feasible and effective. The module can be used as the speed feedback device of the brushless DC motor controller, and the other speed device is not required. It can be used in the speed field of ordinary brushless DC motor.
\end{abstract}

\section{Keywords- BrushlessDCMotors ; Measurement;} Experiment; Parameter; Atmega8515L

\section{INTRODUCTION}

Permanent magnet brushless DC motor has a good speed performance, such as step less speed, wide speed range, high efficiency and so on, which is widely used in underwater robot propeller driven system. Taking into account the simplified design of propulsion machinery structure, signal detector directly by the Holzer motor itself of position and velocity feedback, without adding other location and speed detection devices. The Holzer

\author{
Mao Yongming \\ School of Information \& Control Engineering \\ Shenyang Jianzhu University \\ Shenyang China \\ Liu Chunguang \\ Editorial Department of Journal \\ Shenyang Jianzhu University \\ Shenyang, China \\ Liu Xiyang \\ School of Information \& Control Engineering \\ Shenyang Jianzhu University \\ Shenyang China \\ Wang Bin \\ Northeastern University at Qinhuangdao \\ Qinhuangdao, China
}

signal measurement of speed usually in two ways: one is measured using a Holzer signal, because the single channel signal resolution is low, the feedback cycle is long, usually used in the speed control field; one is the use of three Holzer signal, through logic or algorithms, frequency signal generator 6 times in the way Holzer signal frequency, and then to measure its. The second kind of method is used in literature [1-3], this method enhances the resolution, can be used in the field of low speed control. But these two methods have strict requirements for positioning Holzer sensor in the motor stator circumference, when Holzer sensor in the motor stator circumference positioning error, the adjacent two positive pulse width is inconsistent, can lead to large errors in measurements[4-6].

This paper has made the improvement to the second kinds of methods, this paper introduces an ordinary brushless DC motor (on Holzer sensor is not positioned on the motor stator circumference on the strict requirements of the motor) for the six frequency and direction processing technology, the design of a speed signal by using Holzer function modules, obtained the good control effect.

\section{ACCORDING TO THE BASIC PRINCIPLE OF HOLZER SIGNAL SPEED}

Holzer switch type is used in detector of rotor position of Brushless DC motor using, usually with Holzer 
position signal of motor commutation control[7-8]. After the surgery, Holzer signals from the sensor $\mathrm{Ha}, \mathrm{Hb}, \mathrm{Hc}$ signal is a low frequency pulse three differ by 120 degrees [9-10], the three signals composed of 6 different code in an electric angle of 360 degrees, the formation of six different states, the six codes are $101,100,110,010,011,001$. The rotor is transferred in accordance with the order of change, in the reverse order against the change, so that the three signals with speed and direction information, so that the three signals with speed and direction information. The main idea of this paper is: the speed if logarithmic motor pole N0 motor a lap N0 each group of 6 different states, set a clock frequency is F0 counters, per motor runs into a state, recording the counter value is $\mathrm{Mi}$, the speed of motor.

$$
n_{i}=\frac{60 f_{0}}{m_{i}-m_{i-6 N_{0}}}
$$

Type $\mathrm{i}>=6 \mathrm{~N} 0$. This method has no requirement for locating Holzer element on the motor, speed fluctuation is small, but also can improve the speed feedback signal resolution. This paper describes the design of a functional module by using this method, and to prove the feasibility and reliability of this method through the experiment.

\section{THE FUNCTION MODULE DESIGN}

Design of function module interface circuit

The function module Holzer element velocimetry is implemented by Atmega8515L, the interface circuit schematic diagram shown in Fig. 1

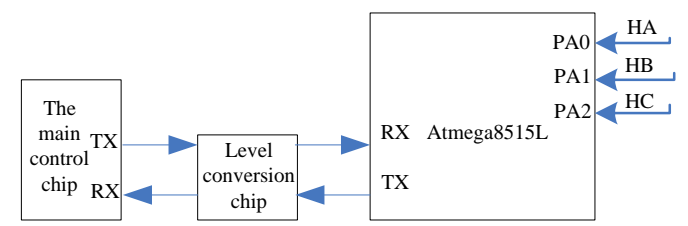

Figure 1. Function module interface circuit schematic diagram

Holzer element output three signals through the shaping is connected to three pin single-chip PA port module through the serial interface, and the main control chip communication. The main control chip is used to complete the motor closed-loop control, generally uses the high-speed processing chip DSP, it is usually supplied by $3.3 \mathrm{~V}$, compatible so between the module and the main control chip to chip level conversion to a level considered. If you use ARM, C8051F040 support 5V logic level chip as the main control chip can skip a level conversion chip, between Atmega8515L and main control chip is connected by the $4.7 \mathrm{~K}$ resistor, also can guarantee the normal work.

Program design of function module

Taking into account the counter overflow, the value is too large and so on, can the formula (1) for deformation, due to

$$
\begin{gathered}
T_{i}=m_{i}-m_{i-1} \\
n_{i}=\frac{60 f_{0}}{\sum_{j=i}^{i-6 N_{0}+1} T_{j}}
\end{gathered}
$$$$
m_{i}-m_{i-6 N_{0}}=\sum_{j=i}^{i-6 N_{0}+1}\left(m_{j}-m_{j-1}\right)
$$

In order to speed up the computation speed must be reduced to and the number of times, a sliding window of the module and algorithm for the $6 \mathrm{~N} 0$ sum into two times the sum, the following processing algorithm of motor speed:

- The definition of a global array A[6N0], the global variable bcnt, atop, sum, the local variable $\mathrm{T}$, and they are initialized to 0 , the current counter readings for $\mathrm{m} 1$;

- Implementation of $\mathrm{T}=\mathrm{mi}-\mathrm{bcnt}$, followed by bcnt=mi, $\quad$ sum $=$ sum $-\mathrm{A}[$ atop] $+\mathrm{T}, \quad \mathrm{A}[$ atop] $=\mathrm{T}$, atop ++ , if atop $=6 \mathrm{~N} 0$, then atop $=0$, the speed of $=60 \mathrm{f} 0 / \mathrm{sum}$;

- In the measurement of motor speed, due to fluctuating speed itself, random disturbance, the measured speed of mean value filter, will achieve better results.

The frequency measurement and identification module program design thought is: to set a timer, the timing time as small as possible, to complete the interrupt program on the line, detection of Holzer signal timing state, accordingly, the program flow diagram of Fig.2(a.b) shows, the state st 0 -st5 respectively and the corresponding code 101100110010011001 .

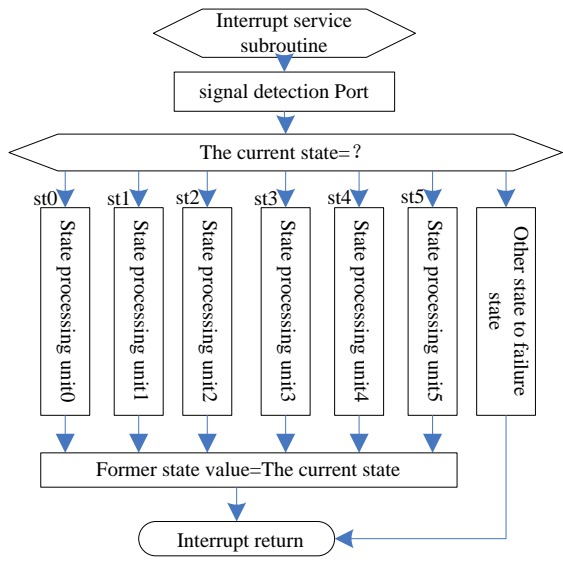

(a) 


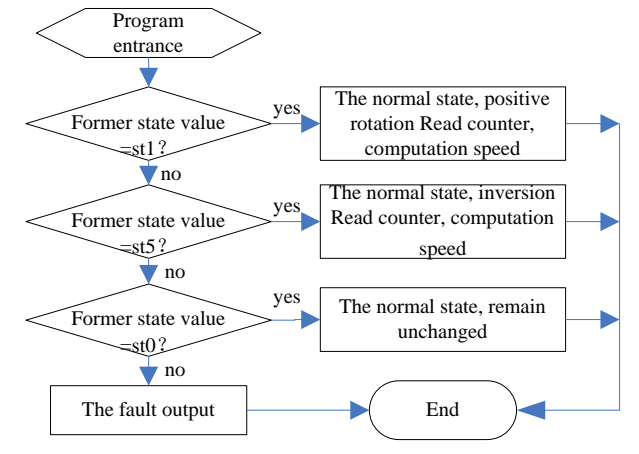

(b)

Figure 2. fault subroutine flow chart (a),State processing unit 0 program flow chart(b)

Module through the serial port to communicate with the main control chip, represented by two bytes speed information, first bits of the direction of rotation of the motor, second said gun fault state, the next 14 bits represents the velocity, so the maximum speed is 16838 rpm, in order to guarantee the normal work of the master and slave communication, the handshake way of working. Specific process is:

- The main control chip to send characters "\#" to indicate the beginning of receiving data module;

- $\quad$ Receives "module \#" after the start, the high-order byte data transmission speed;

- The main control chip receives high byte, transmitting character "1" to the module, said the high byte received, to accept the low order byte;

- $\quad$ Receives "module 1", sending the low order byte;

- The main control chip receives the low order byte, the high-order byte LSB combination for the complete velocity information, and then extracts the velocity magnitude, direction, fault information, communication end.

The benefit of taking this way is: the communication between the two sides interrupt priority is not strictly required, communication information will not be damaged in the process of communication, especially the high byte and the low order byte order.

\section{THE EXPERIMENTAL RESULTS}

Motor parameters in experiments: the rated speed $4000 \mathrm{r} / \mathrm{min}$, power $50 \mathrm{~W}$, the pole number is 4 . Program uses C8051F040 chip as the main control chip, speed feedback function module design. The controller adopts digital double closed loop PID control algorithm, the current loop cycle is $125 \mathrm{us}$, the speed loop cycle is $3 \mathrm{MS}$, get the system steady state speed curve as shown in Fig. 3, shown in Fig.4.

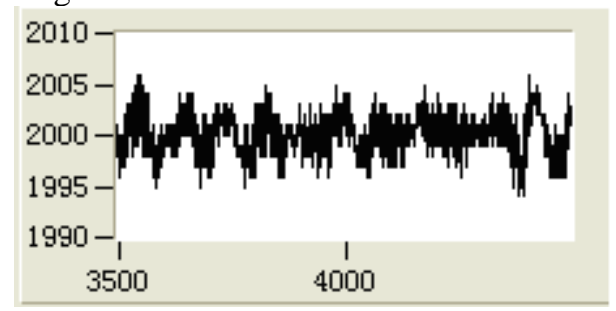

Figure 3. Set the speed for the steady state200r/min

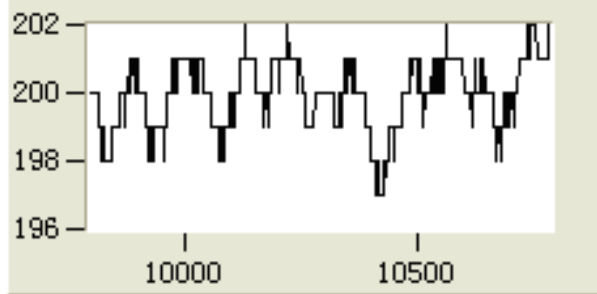

Figure 4. Set the speed for the steady state speed curve of speed curve of $2000 \mathrm{r} / \mathrm{min}$

\section{CONCLUSIONS}

The Holzer signal improved six frequency measurement method based on the design, a special speed module, the experimental results as shown in Figure 4, as shown in Fig.5, the control accuracy reached $0.25 \%$ and $1 \%$, proved the feasibility and reliability of this method, the module can be used as a brushless DC motor speed feedback, without increasing speed measuring device of other.

\section{REFERENCES}

[1] LI Jun-wei, CUI Shi, LI Lian-qiang, REN Chuan-bo, WANG Yu-hai, "Design and Development of Brushless DC Motor Control System," Machinery Design \& Manufacture. Jinan, vol.2, 2013,pp.77-79.

[2] Wang Wei-dong, "Basic principle and control strategy of brushless direct current motor used in electro -hydraulic power steering system,'Machinery Design \& Manufacture. Beijing , vol3, 2010, pp.262-264.

[3] $\mathrm{Xu} \mathrm{Li} \mathrm{-li,} \mathrm{He} \mathrm{Zhi} \mathrm{-qin,} \mathrm{"Simulation} \mathrm{research} \mathrm{on} \mathrm{the} \mathrm{BLDCM}$ control system,'Industrial Control Computer. Beijing , vol24 2011, pp.68-69.

[4] Zhi Liu,Bai-Fen Liu, “Robust Control Strategy for the Speed Control of Brushless DC Motor,"Journal of Harbin Institute of Technology. Ha er bin,vol2,2013,pp.24-25.

[5] Wang Xin,Guo Lili,Ma Lina. Cooperative Spectrum Sensing Algorithm Based on Second User Selection and Random Fores Classification[J].ICIC Express Letters, Part B: Applications,2015, 6(3):845-850

[6] SHEN Jianxin, LI Peng, HAO He, YANG Guang, "Study on Electromagnetic Losses in High-speed Permanent Magnet Brushless Machines-the State of the Art," Proceedings of the CSEE. Beijing,vol.33,2013, pp.62-63.

[7] Shen J, et al, "Sensorless high-speed BLDC machine using hardware-RFO,"The International

Journal for Computation and Mathematics in Electrical and Electronic Engineering, vol33,2012,pp.182-190.

[8] Wang Xin, Huang Kuan, Gao Zhijun. The Primary Users' Signals Recognition Algorithm in Cognitive Radio Networks via KPCA and Random Forest[J].ICIC Express Letters,2015, 9(4):10831088.

[9] Kong Xiaoguang, Wang Fengxiang, Xu Yunlong,“Analysis and calculation of iron losses of high-speed permanent magnet machines,'Electric Machines and Control.Beijing, vol14,2010, pp.26-30.

[10] LI.Jiejia, WU.Chengdong, "Research on Multi-fault Diagnosis Method Based on Integrated Neural Network,"Control Engineering of China.Shenyang,vol19, 2012,pp.407-411. 